\title{
Investigation of Genetic Disturbances in Oxygen Sensing and Erythropoietin Signaling Pathways in Cases of Idiopathic Erythrocytosis
}

\author{
Carla Luana Dinardo, ${ }^{1}$ Paulo Caleb Junior Lima Santos, ${ }^{1}$ Isolmar Tadeu Schettert, ${ }^{1,2}$ \\ Renata Alonso Gadi Soares, ${ }^{1}$ Jose Eduardo Krieger, ${ }^{1}$ and Alexandre Costa Pereira ${ }^{1}$ \\ ${ }^{1}$ Laboratory of Genetics and Molecular Cardiology, Heart Institute, University of São Paulo Medical School, \\ Avenue Dr. Enéas de Carvalho Aguiar, 44 Cerqueira César, 05403-000 São Paulo, SP, Brazil \\ ${ }^{2}$ Novo Atibaia Hospital, 05403-000 São Paulo, SP, Brazil \\ Correspondence should be addressed to Paulo Caleb Junior Lima Santos; pacaleb@usp.br
}

Received 30 August 2013; Revised 11 November 2013; Accepted 13 November 2013

Academic Editor: Michael Walter

Copyright ( 2013 Carla Luana Dinardo et al. This is an open access article distributed under the Creative Commons Attribution License, which permits unrestricted use, distribution, and reproduction in any medium, provided the original work is properly cited.

Background. Idiopathic erythrocytosis is the term reserved for cases with unexplained origins of abnormally increased hemoglobin after initial investigation. Extensive molecular investigation of genes associated with oxygen sensing and erythropoietin signaling pathways, in those cases, usually involves sequencing all of their exons and it may be time consuming. Aim. To perform a strategy for molecular investigation of patients with idiopathic erythrocytosis regarding oxygen sensing and erythropoietin signaling pathways. Methods. Samples of patients with idiopathic erythrocytosis were evaluated for the EPOR, VHL, PHD2, and HIF-2 $\alpha$ genes using bidirectional sequencing of their hotspots. Results. One case was associated with HIF- $2 \alpha$ mutation. Sequencing did not identify any pathogenic mutation in 4 of 5 cases studied in any of the studied genes. Three known nonpathogenic polymorphisms were found (VHL p.P25L, rs35460768; HIF-2 $\alpha$ p.N636N, rs35606117; HIF-2 $\alpha$ p.P579P, rs184760160). Conclusion. Extensive molecular investigation of cases considered as idiopathic erythrocytosis does not frequently change the treatment of the patient. However, we propose a complementary molecular investigation of those cases comprising genes associated with erythrocytosis phenotype to meet both academic and genetic counseling purposes.

\section{Introduction}

Absolute erythrocytosis should be suspected when hemoglobin $(\mathrm{Hb})$ is greater than $16.5 \mathrm{~g} / \mathrm{dL}$ in females or $18.0 \mathrm{~g} / \mathrm{dL}$ in males [1] and defines a status of elevated hemoglobin. Causes of erythrocytosis can be divided into two groups: primary, in which there is an intrinsic problem in bone marrow driving abnormal erythropoiesis, or secondary, in which there is an event outside bone marrow leading to an abnormal production of erythrocytes [1]. Both groups can be further classified into acquired or congenital. Within the group of primary erythrocytosis, erythropoietin receptor (EPOR) mutation (congenital) and polycythemia vera (PV, acquired) stand out. Within the group of secondary erythrocytosis, there are multiple acquired causes (hypoxia, EPO pathologic production, and drugs) and a restricted subgroup of congenital causes (VHL, PHD2, and HIF-2 $\alpha$ mutations; high oxygen-affinity hemoglobin; and bisphosphoglycerate mutase deficiency) (Figure 1) [1-3].

Idiopathic erythrocytosis is the term reserved for the cases with an unexplained cause of erythrocytosis after initial investigation. Even though this classification is currently applied in clinical practice, it lacks precise criteria. Usually, a patient that neither could be classified as PV nor presents with an acquired cause of erythrocytosis is labeled as having idiopathic erythrocytosis. Further investigation is of questionable clinical value. Cases with a suspicion of congenital causes may be associated with disturbances of oxygen sensing and erythropoietin signaling pathways. 
Erythrocytosis without acquired secondary causes
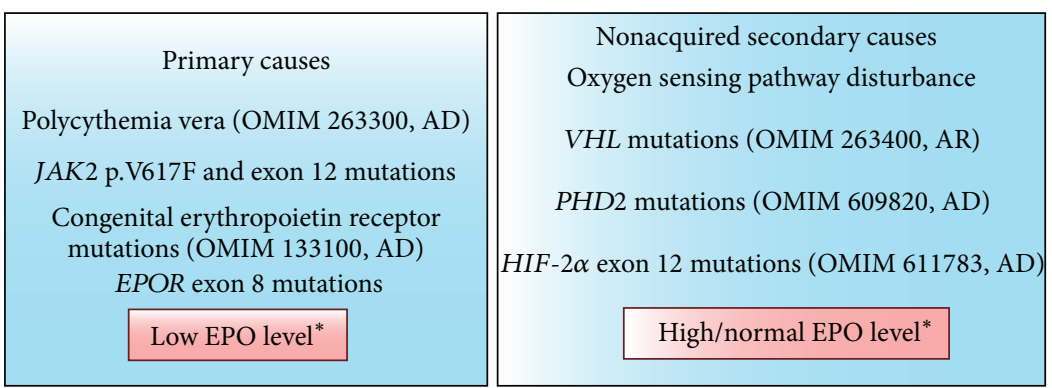

FIGURE 1: Representation of diagnostic strategy for patients with erythrocytosis without acquired secondary causes. The most common example of primary erythrocytosis is polycythemia vera (PV). Notably, the majority of idiopathic erythrocytosis patients cannot be classified into the OMIM categories, nor do they have a characterized molecular defect. Consequently, patients with established but unexplained erythrocytosis warrant further investigation [1]. AD: autosomal dominant inheritance, AR: autosomal recessive inheritance. ${ }^{*}$ EPO levels can suggest origins of this disorder.

In this scenario, we propose a molecular strategy for evaluating genes regarding oxygen sensing and erythropoietin signaling pathways in cases of idiopathic erythrocytosis with suspected congenital causes.

\section{Methods}

Five Brazilian patients with suspected idiopathic erythrocytosis were selected at Novo Atibaia Hospital, SP, Brazil, and Heart Institute, SP, Brazil. The study protocol was approved by the involved Institutional Ethics Committees, and written informed consent was obtained from all patients. General characteristics of patients were obtained through an interview.

Patients enrolled fulfilled the following inclusion criteria:

(1) documented persistent erythrocytosis $(16.5 \mathrm{~g} / \mathrm{dL}$ in females or $18.0 \mathrm{~g} / \mathrm{dL}$ in males);

(2) JAK2 p.V617F negative and normal JAK2 exon 12 sequencing;

(3) arterial blood gas analysis (including p50) within normal range;

(4) biochemical analysis within normal range;

(5) bone marrow biopsy normal or revealing erythroid expansion;

(6) absence of symptoms of obstructive sleep apnea or normal polysomnography;

(7) absence of masses in abdominal tomography or abdominal ultrasound.

Genomic DNA was isolated from peripheral blood leukocytes by a salting-out method. Coding sequences of EPOR exon $8, V H L$ exons $1-3$, PHD2 exons $1-5$, and HIF- $2 \alpha$ exon 12 were amplified by a polymerase chain reaction (PCR) using the previously described primer sequences $[4,5]$. Exons were selected based on literature review and consisted of known hotspots. PCR products were purified using ExoSAPIT reagent (GE Healthcare, NJ, USA) and were bidirectionally sequenced (ABI Terminator Sequencing Kit and ABI 3500XL Sequencer, Applied Biosystems, Foster City, CA, USA).

\section{Results and Discussion}

We identified a novel mutation in HIF- $2 \alpha$ exon 12 in one patient, using the suggested investigation, which was published elsewhere and comprised a strong family history of arterial thromboembolic events [6]. The others had EPO levels within normal range and absence of family history of erythrocytosis or thromboembolism and sequencing of EPOR exon 8, VHL exons $1-3$, PHD2 exons $1-5$, and HIF$2 \alpha$ exon 12 did not identify any mutation. Three known nonpathogenic polymorphisms were found: VHL c.C74T polymorphism (p.P25L, rs35460768, exon 1, and heterozygous genotype), HIF-2 $\alpha$ c.T1908C polymorphism (p.N636N, rs35606117, exon 12, and heterozygous genotype), and HIF-2 $\alpha$ c.G1737A polymorphism (p.P579P, rs184760160, exon 12, and heterozygous genotype). Results were ready after less than one week after blood samples were obtained. Phlebotomies and acetylsalicylic acid use (100 mg/day) were prescribed to all patients, with normalization of hemoglobin and hematocrit values and favorable outcome.

The investigation of a patient with absolute erythrocytosis involves a detailed clinical history and physical examination as well as an already standardized laboratory evaluation. In cases of absolute erythrocytosis without any acquired secondary cause, $P V$ should be ruled out at first and considering the current dissemination of molecular techniques designed to detect p.V617F JAK2 mutation, most medical centers manage to diagnose their $P V$ cases without great difficulties. Sequencing JAK2 exon 12, the next step if JAK2 p.V617F mutation is absent, is quite more challenging and easily forgotten during the laboratory investigation [7]. Besides $J A K 2$ exon sequencing, detection of mutation on this region can be also performed for high resolution melting (HRM) analysis as shown by previous studies [8-10].

Many physicians would not go further after this initial investigation, since there will be no significant changes 
regarding patients' clinical management. However, when there is a suspicion of a congenital cause behind a case of idiopathic erythrocytosis, a complementary molecular investigation regarding the most important causes may become important, especially in terms of genetic family counseling. According to current medical literature, disturbances involving oxygen sensing pathway, presence of high oxygen affinity hemoglobin, and mutations in erythropoietin receptor (EPOR) stand out in the group of congenital causes of erythrocytosis.

In order to avoid extensive sequencing strategies comprising all exons of all genes involved in oxygen sensing and erythropoietin signaling pathways, we validated in this study a protocol in which the most important genes of both pathways are evaluated and, in those two chosen genes, only hotspots exons are sequenced [5,11-13]. Our protocol consists in sequencing EPOR exon 8, VHL exons 1-3, PHD2 exons 15 , and HIF2- $\alpha$ exon 12 . For a more structured investigation, we suggest sequencing EPOR exon 8 for cases of idiopathic erythrocytosis where EPO level is low and sequencing $V H L$ exons 1-3, PHD2 exons 1-5, and HIF2- $\alpha$ exon 12 for cases of idiopathic erythrocytosis where EPO level is normal or high (Figure 1).

In our presented validation, family history of erythrocytosis/arterial thromboembolic events was the most important anamnesis data, as it was absent in the cases where no mutations were found but present in the mutant case. One drawback of our suggested sequencing strategy is that some nonpathogenic polymorphisms may be identified, but bioinformatics programs can easily overcome this obstacle. A comprehensive database concerning molecular and laboratory data of congenital erythrocytosis patients is provided by the EU Congenital Erythrocytosis (ECE-C) Consortium (http://www.erythrocytosis.org). In addition, it represents a useful tool when evaluating if a certain discovered genetic alteration has already been described as pathogenic [14, 15]. Another main database about myeloproliferative diseases is the European Cooperation in Science and Technology (http://www.mpneuronet.eu). An available reference, "Clinical utility gene card for familial erythrocytosis," shows genetic, clinical, and practical aspects about erythrocytosis [16]. Studies indicated an undetermined proportion of positive genetic tests if the disease is present. But, they suggest that $<50 \%$ of cases with erythrocytosis and positive family history are likely positive for one of the associated mutations $[1,16]$.

The ECE-C has recently described their strategy for evaluating patients with congenital erythrocytosis [15]. Our suggested strategy is similar to theirs in the case of CE patients with low EPO level, as sequencing EPOR exon 8 is suggested. In the case of $\mathrm{CE}$ patients with normal or high EPO level, the ECE-C organizes their sequencing strategy based on the presence or absence of family history. In our guideline, we suggested sequencing $V H L$ exons 1-3, PHD2 exons 1-5, and HIF2- $\alpha$ exon 12 irrespective of family history, mainly because of the high prevalence of cardiovascular diseases, which hampers the distinction between real/casual antecedents and may impair the investigation and also because of the logistics facility of performing all sequencing reactions together, considering the low amount of samples.
In our suggested investigation strategy, we do not consider routinely sequencing other less commonly affected genes when the initial approach does not give a positive result. This is based on the fact that our CE patients with a negative investigation presented a favorable outcome after the beginning of the phlebotomy routine and also on our aims of providing a rational, cost-effective, and non-time-consuming tool. However, when a mutation is found, patients can be properly followed and evaluated regarding complications that may not be directly related to the level of hemoglobin, as it has already been previously described for mutation in HIF gene [17] and for the VHL gene $[18,19]$.

Nowadays, to perform next generation sequencing (NGS) for new candidate genes poses a promising tool to discover new mutations in $\mathrm{CE}$ patients. However, considering the costs and time consumption of this strategy, it should still be reserved for only some centers or academic purposes. New genes and mutations discovered using this technology should be evaluated regarding pathogenicity to, then, be incorporated to erythrocytosis-related genes group. In the cases with strong family history, to expand investigation and eventually to perform NGS may be advised, as the odd of discovering new mutations is higher.

\section{Conclusion}

We propose the investigation of disturbances of oxygen sensing and erythropoietin signaling pathways in cases of idiopathic erythrocytosis with positive family history of erythrocytosis/thromboembolism. Our suggested sequencing protocol is economically feasible and sensitive, and it might help both academic and genetic counseling purposes.

\section{Conflict of Interests}

The authors declare that they have no conflict of interests.

\section{Acknowledgments}

PCJL Santos is recipient of Proc.2013/09295-3, Proc.2013/ 20614-3, and Proc.2010/17881-1 from FAPESP, Brazil. The authors also thank the patients who participated in the study. The technical assistance of the Laboratory of Genetics and Molecular Cardiology group, Heart Institute group, is gratefully acknowledged.

\section{References}

[1] F. S. Lee and M. J. Percy, "The HIF pathway and erythrocytosis," Annual Review of Pathology: Mechanisms of Disease, vol. 6, pp. 165-192, 2011.

[2] M. F. McMullin, "The classification and diagnosis of erythrocytosis," International Journal of Laboratory Hematology, vol. 30, no. 6, pp. 447-459, 2008.

[3] D. J. Weatherall, "Polycythemia resulting from abnormal hemoglobins," The New England Journal of Medicine, vol. 280, no. 11, pp. 604-606, 1969. 
[4] M. J. Percy, P. A. Beer, G. Campbell et al., "Novel exon 12 mutations in the HIF2A gene associated with erythrocytosis," Blood, vol. 111, no. 11, pp. 5400-5402, 2008.

[5] M. J. Percy, P. W. Furlow, P. A. Beer, T. R. J. Lappin, M. F. McMullin, and F. S. Lee, "A novel erythrocytosis-associated PHD2 mutation suggests the location of a HIF binding groove," Blood, vol. 110, no. 6, pp. 2193-2196, 2007.

[6] M. J. Percy, Y. J. Chung, C. Harrison et al., "Two new mutations in the HIF2A gene associated with erythrocytosis," American Journal of Hematology, vol. 87, no. 4, pp. 439-442, 2012.

[7] M. J. Percy, L. M. Scott, W. N. Erber et al., "The frequency of JAK2 exon 12 mutations in idiopathic erythrocytosis patients with low serum erythropoietin levels," Haematologica, vol. 92, no. 12, pp. 1607-1614, 2007.

[8] R. H. Nussenzveig, T. Burjanivova, M. E. Salama et al., "Detection of JAK2 mutations in paraffin marrow biopsies by high resolution melting analysis: identification of L611S alone and in cis with V617F in polycythemia vera," Leukemia \& Lymphoma, vol. 53, no. 12, pp. 2479-2486, 2012.

[9] P. C. Junior Lima Santos, R. A. Gadi Soares, J. E. Krieger, E. M. Guerra-Shinohara, and A. C. Pereira, "Genotyping of the hemochromatosis HFE p.H63D and p.C282Y mutations by high-resolution melting with the Rotor-Gene 6000 instrument," Clinical Chemistry and Laboratory Medicine, vol. 49, no. 10, pp. 1633-1636, 2011.

[10] S. Carillo, L. Henry, E. Lippert et al., "Nested high-resolution melting curve analysis: a highly sensitive, reliable, and simple method for detection of Jak2 exon 12 mutations-clinical relevance in the monitoring of polycythemia," Journal of Molecular Diagnostics, vol. 13, no. 3, pp. 263-270, 2011.

[11] M. Al-Sheikh, E. Mazurier, B. Gardie et al., "A study of 36 unrelated cases with pure erythrocytosis revealed three new mutations in the erythropoietin receptor gene," Haematologica, vol. 93, no. 7, pp. 1072-1075, 2008.

[12] K. O'Rourke, D. J. Fairbairn, K. A. Jackson, K. L. Morris, S.-K. Tey, and G. A. Kennedy, "A novel mutation of the erythropoietin receptor gene associated with primary familial and congenital polycythaemia," International Journal of Hematology, vol. 93, no. 4, pp. 542-544, 2011.

[13] M. J. Percy, "Genetically heterogeneous origins of idiopathic erythrocytosis," Hematology, vol. 12, no. 2, pp. 131-139, 2007.

[14] H. Cario, M. F. McMullin, C. Bento et al., "Erythrocytosis in children and adolescents-classification, characterization, and consensus recommendations for the diagnostic approach," Pediatric Blood \& Cancer, vol. 60, no. 11, pp. 1734-1738, 2013.

[15] C. Bento, M. J. Percy, B. Gardie et al., "Genetic basis of congenital erythrocytosis: mutation update and online databases," Human Mutation, 2013.

[16] K. Hussein, M. Percy, and M. F. McMullin, "Clinical utility gene card for: familial erythrocytosis," European Journal of Human Genetics, vol. 20, no. 5, 2012.

[17] E. R. Woodward, K. Wall, J. Forsyth, F. Macdonald, and E. R. Maher, "VHL mutation analysis in patients with isolated central nervous system haemangioblastoma," Brain, vol. 130, part 3, pp. 836-842, 2007.

[18] V. R. Gordeuk and J. T. Prchal, "Vascular complications in Chuvash polycythemia," Seminars in Thrombosis and Hemostasis, vol. 32, no. 3, pp. 289-294, 2006.

[19] V. R. Gordeuk, A. I. Sergueeva, G. Y. Miasnikova et al., "Congenital disorder of oxygen sensing: association of the homozygous Chuvash polycythemia VHL mutation with thrombosis and vascular abnormalities but not tumors," Blood, vol. 103, no. 10, pp. 3924-3932, 2004. 

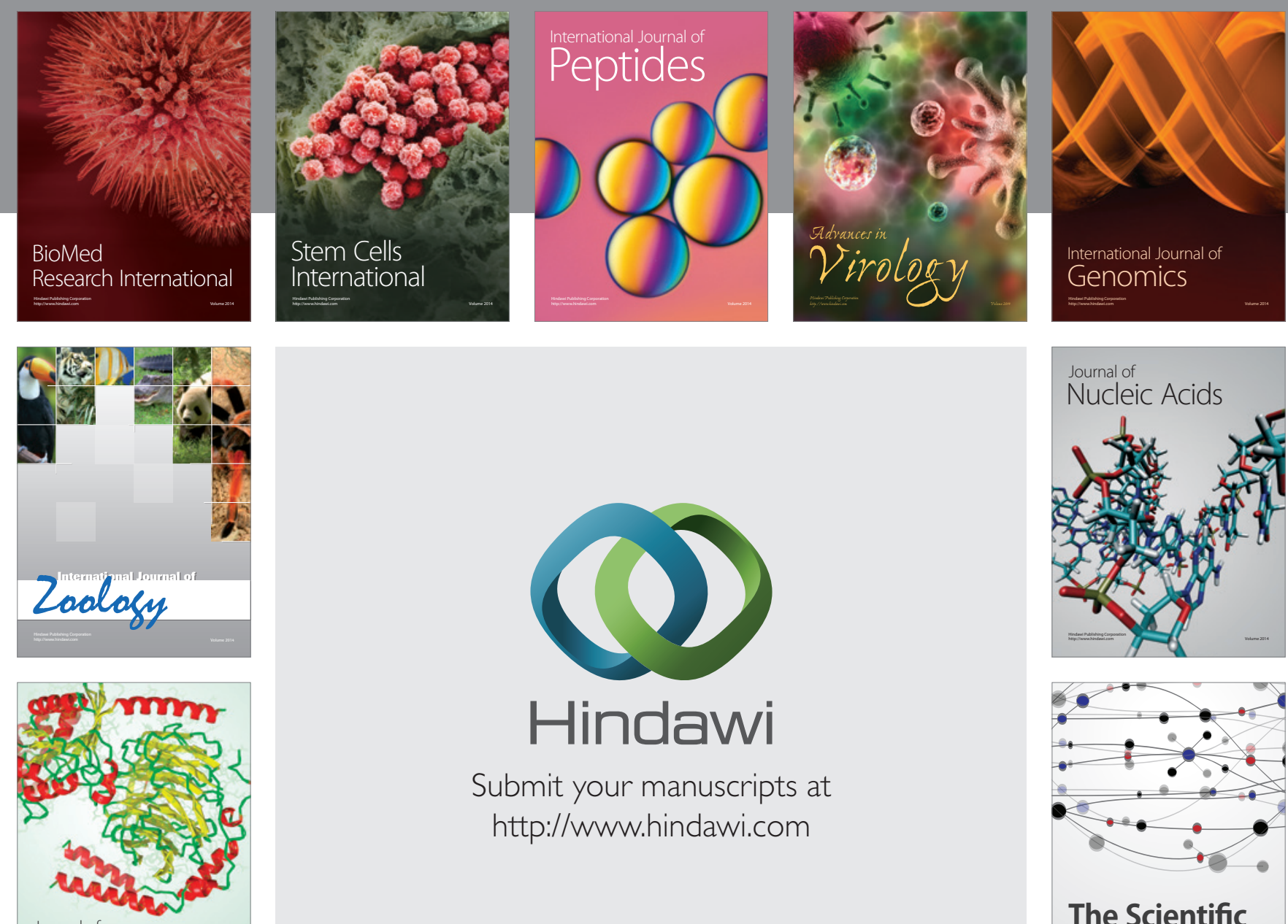

Submit your manuscripts at

http://www.hindawi.com

Journal of
Signal Transduction
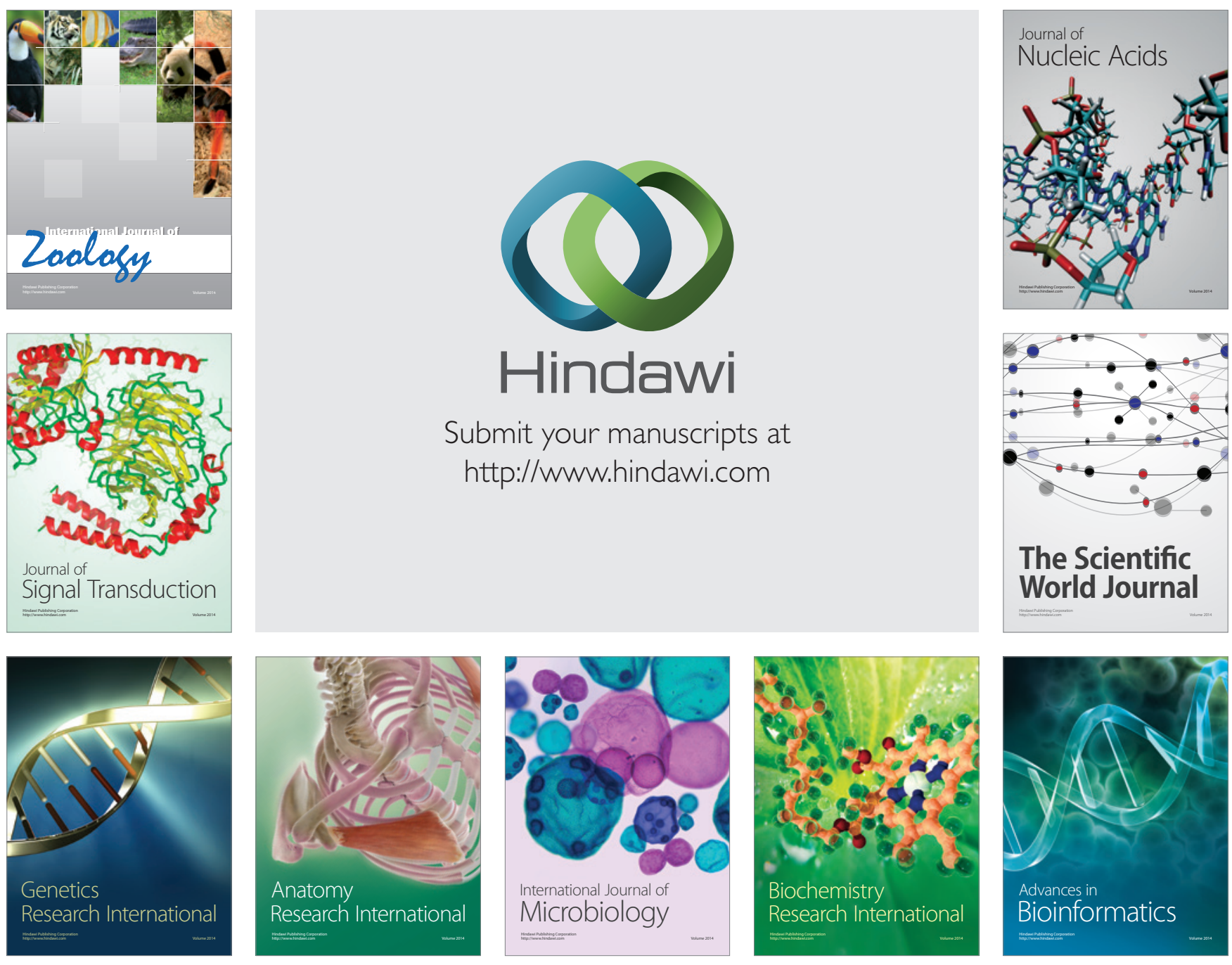

The Scientific World Journal
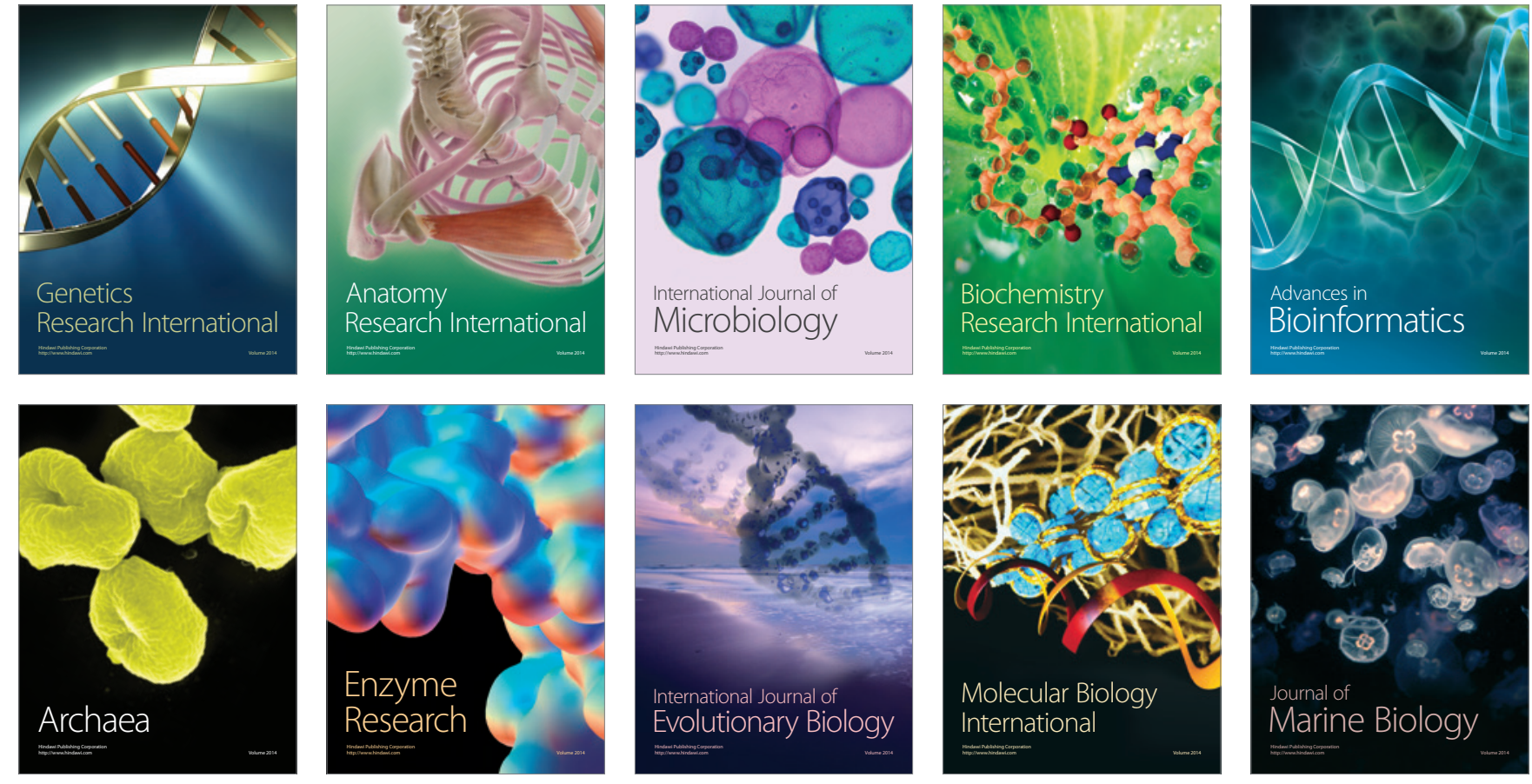